\title{
Investigations on Modeling and Simulation of Printed Folded Dipole Antenna
}

\author{
Prabir Banerjee ${ }^{1}$ and Tulshi Bezboruah ${ }^{2}$ \\ ${ }^{1}$ Department of Physics, Jagiroad College, Jagiroad 782410, Morigaon, Assam, INDIA \\ Tel: +91-367-8242308(O) \\ ${ }^{2}$ Department of Electronics \& Communication Technology, Gauhati University, Guwahati- \\ 781014, Assam, INDIA \\ Tel: +91361-2671262(O); Fax: +91-361-2700311(O) \\ prabirjrd1962@gmail.com1,zbt_gu@yahoo.co.in ${ }^{2}$
}

\begin{abstract}
We have modeled and simulate asymmetric microstrip folded dipole antenna on substrate materials and study the antenna performances. The conventional wire dipole antenna configurations are converted into corresponding microstrip version. The complementary nature of parallel coplanar strip line and waveguide models have been used to model the dipole antenna. The transmission line parameters namely, even-odd mode effective dielectric constant and characteristic impedance have been analyzed. We study the effect of dielectric material on radiation characteristics of the printed folded dipole antenna, such as effective dielectric constant, characteristics impedance, input impedance and bandwidth. The antenna shows bandwidth improvement below $3 \mathrm{GHz}$ frequency with asymmetric form of strip folded dipole.
\end{abstract}

Keywords: Bandwidth, Characteristics impedance, Folded Dipole antenna, Input impedance, Microstrip dipole antenna, Waveguide

\section{Introduction}

A compact size antenna design with wide bandwidth (BW) characteristics is the major challenge in communication system. Another factor which over shadow its performance is its narrow BW and low efficiency when the size is reduced. First successful design of printed dipole was reported in 1987 by D. Edward et al. [1]. With proper design, printed antennas are most suitable for varieties of applications due to their planar structure and compatibility with the printed circuit fabrication techniques [7]. Printed antennas may be classified into two main categories on the basis of width to length ratio, namely: (a) Microstrip antenna and (b) Microstrip dipole antenna [7]. An antenna with narrow strip width less than $\mathbf{0 . 0 5} \boldsymbol{\lambda}_{\mathbf{0}}$ $\left(\lambda_{0}=\right.$ free space wavelength) is called microstrip dipole [7]. Printed dipoles are sometimes used in array application, because they occupy less space compared to conventional microstrip antennas.

The strip folded dipole antenna fabricated on dielectric substrate gives wider BW and provide good matching characteristics due to its large input impedance compared to single element strip dipole.

This antenna serves as a step-up impedance transformer of the single-element impedance, when the spacing(s) between the two larger sides is very small (usually $\boldsymbol{s}<\mathbf{0 . 0 5} \lambda$ ) [9]. When arm length $\mathbf{L}=\boldsymbol{\lambda / 2}$, the antenna is resonant and its impedance is about $300 \mathrm{ohms}$ and can be used for connections to twin-lead transmission lines [9]. 
Our study mainly focused on transition from wire dipole to corresponding printed folded dipole in asymmetric form. We simulate the key antenna parameters with MATLAB simulation software. We investigate the same by considering the parameters namely: (i) input impedance, (ii) BW, (iii) even and odd mode effective dielectric constant and (iv) characteristic impedance of coplanar strips (CPS) in which the folded dipole is a section and (v) ) characteristic impedance Waveguide mode.

\subsection{Related Works}

In the year 1980, G. A. Thiele, E. P. Ekelman, Jr., and L. W. Henderson [2] proposed folded dipole of circular cross section and its equivalent transmission-line and antenna mode models and validated the model by numerical method such as Moment Method.

In the year 1985 and 1986, R. W. Lampe [3, 4] proposed design formulas for asymmetric coplanar strip folded dipole in homogeneous medium.

C. A. Balanis (2005) [9] proposed step by step design procedure of wired folded dipole antenna including expressions for equivalent radius, input impedance, characteristic impedance, current division factor and matching networks.

In the year 2007, Chen-To Tai, Stuart A. Long, proposed design formula similar to [12] except slightly different current division formula for element spacing compared to the radius of the wired folded dipole antenna.

In the year 2007, J. K. Raines [11] developed a mathematical model of the wired folded antenna with arbitrary impedance loaded at both top and base which is applicable to both long $\left(\mathbf{L}>\boldsymbol{\lambda}_{\mathbf{0}}\right)$ and $\operatorname{short}\left(\mathbf{L}<\boldsymbol{\lambda}_{\mathbf{0}}\right)$ antennas.

In the year 2010, H.J. Visser [5] modified the design formulas of asymmetric strip folded dipole in homogeneous medium proposed by R.W. Lampe [3, 4] which can be applied to antenna on dielectric substrate. They also employed correction factors for the dipole length and equivalent radius in homogeneous medium.

In the year 2013, Guang Hua, Chen Yang, Ping Lu, Hou-Xing Zhou, and Wei Hong [6] proposed an odd-even mode design procedure to calculate input impedance for asymmetric strip folded dipole with smaller width of center conductor.

Different authors [5-6, 9, 11-12] proposed current division factor $(\boldsymbol{\alpha})$ for wired folded dipole and strip dipole model. We transformed each model into corresponding strip dipole version necessary to compare the performance of the antenna with different values of $\boldsymbol{\alpha}$.

\section{Design Considerations}

In case of the free-space cylindrical dipole, the electric field between the gaps in the strip of a printed dipole is along the length of the dipole [7]. This distribution is different from that in a patch antenna in which the dominant electric field component is normal to the substrate [7]. Therefore, it is not possible to feed a printed dipole directly with a microstrip line where the major electric field component is normal to the substrate [7]. According to [7], the possible feed structure for printed dipole is coplanar strips (CPS), which is compatible, physically and electrically, with the coplanar strips printed dipole. The asymmetric strip folded dipole can be designed and can be mounted on a dielectric substrate of thickness $\mathbf{h}$. The relative dielectric constant may be in the range of $2.32 \leq \epsilon_{r} \leq \mathbf{6 . 1 5}$. If the antenna is constructed without ground plane, its radiation pattern is likely to be similar to the dipole of the same length $\mathrm{L}$. The structure provides almost four times lager input impedance than that of a single dipole for $\mathbf{L} \leq \boldsymbol{\lambda} / \mathbf{2}$ [9]. The length of dipole antennas are generally in the range $\boldsymbol{\lambda} / \mathbf{4}$ $\leq \mathrm{L} \leq \boldsymbol{\lambda} / \mathbf{2}$ for better performance [9]. Length $\mathrm{L}$ is slightly less than $\lambda / \mathbf{2}$ to account for fringing length extension and to make the input impedance real [9]. The separation between fed-line 
connecting the antenna should satisfy $\mathbf{d}<\mathbf{0 . 0 0 2} \lambda$ [7]. The separation distance (s) between the two strip transmission lines of the folded dipole should not exceed $\mathbf{0 . 0 5} \lambda$ [7].

\section{The Model}

The configuration of the proposed folded dipole antenna in asymmetric form is shown in Figure 1. The antenna is constructed by using microstrip folded dipoles connected with a feed line on a bottom substrate having a thickness $\mathbf{h}$. The folded dipole has better BW characteristics than a single dipole of the same size [9]. Its geometrical arrangement is somewhat like a small side by side stub line which tries to nullify the reactance of a nonresonant lone dipole antenna [9]. According to the traditional analysis method [9], the excitation of a folded dipole is decomposed into two fundamental modes: (i) the transmission line mode and (ii) antenna radiation mode. The folded dipole for T-match can also be modeled by transmission line and antenna modes [9]. The total current at the input terminals is divided between the two conductors in a way that depends on the relative width of the two conductors and the spacing between them [9]. Since the two conductors are not in general of the same width, the antenna mode current division is not unity. So a current division factor is assigned which also applies to the voltage division of the transmission-line mode [9]. The design is performed in two steps. The first step is to calculate the input impedance of asymmetric strip line in transmission mode. The second step is to calculate the input impedance of folded dipole in antenna mode and then using the traditional method the equivalent input impedance is obtained [9].

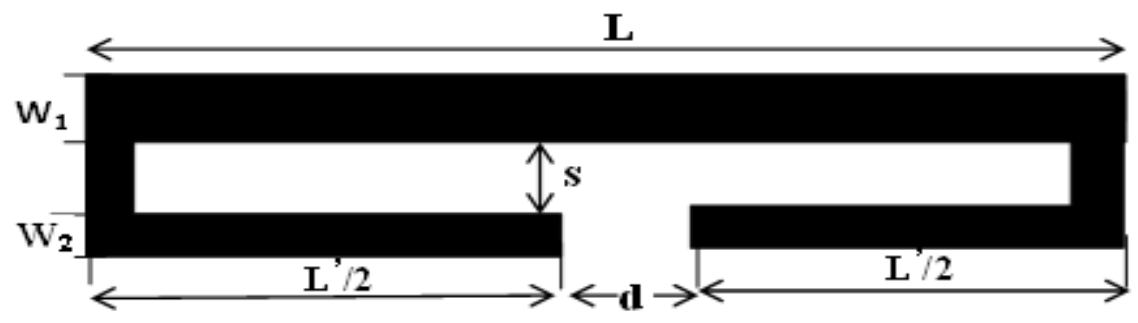

Figure 1. Top View of Asymmetric Strip Folded Dipole

\subsection{Input Impedance}

The feed port input impedance of folded dipole is given by [9]:

$$
Z_{\text {in }}=\frac{2 Z_{t}\left[(1+\alpha)^{2} Z_{A}\right]}{2 Z_{t}+(1+\alpha)^{2} Z_{A}}
$$

Where $\boldsymbol{Z}_{\boldsymbol{A}}$ is the input impedance of strip dipole antenna with length $\boldsymbol{L}$ and width $\mathbf{W}_{1}$ in antenna mode, $\boldsymbol{Z}_{\boldsymbol{t}}=\boldsymbol{j} \boldsymbol{Z}_{\boldsymbol{c}} \tan \left(\boldsymbol{k} \boldsymbol{L}^{\prime} / \mathbf{2}\right)$ is input impedance in transmission line mode, $\boldsymbol{Z}_{\boldsymbol{c}}$ is characteristic impedance of couple line defined in eqn.(4) or eqn.(6), $\boldsymbol{k}$ is wave number and $\boldsymbol{\alpha}$ is current division factor.

The current division factor for non-uniform dipole radius transformed into equivalent radius for very thin strip dipole can be expressed as [9]:

$$
\alpha=\cosh ^{-1}\left(\frac{v^{2}-u^{2}+1}{2 v}\right) / \cosh ^{-1}\left(\frac{v^{2}+u^{2}-1}{2 v u}\right)
$$

(1a)

With $u=W_{1} / W_{2}, v=4 S / W_{2}$

Current division factor reported in [6] is given by: 


$$
\alpha_{1}=\left(\ln \left[4 \mathrm{D}+\sqrt{\mathrm{D}_{1}}\right]-\ln \mathrm{W}_{1}\right) /\left(\ln \left[4 \mathrm{D}+\sqrt{\mathrm{D}_{2}}\right]-\ln \mathrm{W}_{2}\right)
$$

(1b)

With $\mathrm{D}=s / 2+0.25 W_{1}+0.25 W_{2}, D_{1}^{2}=4 D^{2}-0.25 W_{1}^{2}, D_{2}^{2}=4 D^{2}-0.25 W_{2}^{2}$

When $\mathbf{W}_{1}$ and $\mathbf{W}_{\mathbf{2}}$ are small compared with the spacing (s), the current division factor proposed in [12] may be modified from circular cross section to strip folded dipole with the concept of equivalent radius given in [9] can be expressed as:

$$
\alpha_{2}=\ln \left(4 \mathrm{~s} / W_{2} / \ln \left(4 \mathrm{~s} / \mathrm{W}_{1}\right)\right)
$$

(1c)

Another value of current division factor reported in [5] is given by:

$$
\alpha_{3}=\left(\ln \left(4 C+2 \sqrt{A}-\ln \left(W_{2}\right)\right) /\left(\ln \left(4 C+2 \sqrt{A_{1}}-\ln \left(W_{1}\right)\right)\right.\right.
$$

(1d)

With $\mathrm{A}=(2 C)^{2}-\left(W_{2} / 2\right)^{2}, A_{1}=(2 C)^{2}-\left(W_{1} / 2\right)^{2}$ and $2 \mathrm{C}=\mathrm{s}+W_{1} / 2+W_{2} / 2$

Further current division factor proposed in [11] modified for strip folded dipole as:

$\alpha_{4}=\ln \left(0.25 W_{2} /\left(s+0.25 W_{1}\right) / \ln \left(0.25 W_{1} /\left(s+0.25 W_{2}\right)\right.\right.$

(1e)

The input impedance $\boldsymbol{Z}_{\text {in }}$ of the asymmetric strip folded dipole of eqn.(1) can be expressed as [9]:

$$
Z_{\text {in }}=R_{\text {in }}+j X_{\text {in }}
$$

(2)

Where $\boldsymbol{R}_{\boldsymbol{i n}}$ and $\boldsymbol{X}_{\boldsymbol{i n}}$ are the real and imaginary part of input impedance of strip dipole antenna.

The reflection coefficient $\boldsymbol{\Gamma}$ and Voltage Standing Wave Ratio (VSWR) can be expressed as [9]:

$$
\begin{aligned}
& \Gamma=\left(R_{i n}-Z_{c}\right) /\left(R_{\text {in }}+Z_{c}\right) \\
& (3) \\
& \operatorname{VSWR}=(1+\Gamma) /(1-\Gamma)
\end{aligned}
$$

(3a)

\subsection{Characteristic Impedance}

At very high frequency operation, substrate height plays a crucial role in accurate formulation of effective dielectric constant. When substrate height is not very small compared with the in the dielectric, the quasi-static expressions may be inadequate [7]. In case of a digital signal with short rise time propagates along a microstrip line, the signal can be assumed to contain a number of high-frequency harmonics [10]. In such a situation, an accurate dispersion model for propagation along microstrip lines is required [10]. The characteristic impedance can be determined by odd-even method to account for dispersion. An asymmetric folded dipole in antenna mode can be viewed as symmetric two-port microwave network. We have proposed: (a) Coplanar Waveguide models and (b) Coplanar Strip model for accurate determination of characteristics impedance and effective dielectric constant.

3.2.1. Coplanar Waveguide Model: The Coplanar waveguide model (CPW), printed on a finite-thickness dielectric substrate is shown in Figure 2. The width of the dielectric substrate is assumed to be finite. The quasi-static expression for characteristic impedance and effective dielectric constant of this transmission line are given by [7]: 
(4)

$$
Z_{c}=30 \pi K\left(k^{\prime}\right) / K(k) \sqrt{\epsilon_{r e}}
$$

$$
\epsilon_{r e}=\mathbf{1}+\mathbf{0 . 5}\left(\in_{r}-\mathbf{1}\right)\left(K\left(k^{\prime}\right) K\left(k_{1}\right)\right) /\left(K(k) K\left(k_{1}^{\prime}\right)\right)
$$

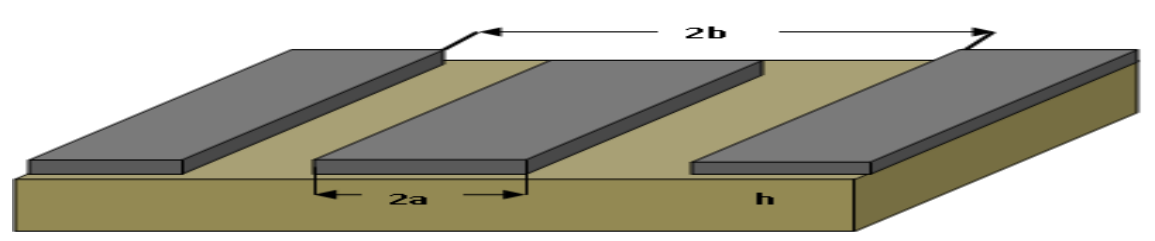

Figure 2. CPW Model of Transmission Lines with $2 \mathrm{~b}=W_{1}+W_{2}+\mathrm{S}$ separated by Distance S=2a Representing Equivalent Printed Folded Dipole

In the above expressions (eqn.(4) and eqn.(5)) $\mathbf{K}$ is complete elliptical integral of first kind. An approximate expression for $\boldsymbol{K}(\boldsymbol{k}) / \boldsymbol{K}\left(\boldsymbol{k}^{\prime}\right)$ which is accurate to $8 \mathrm{ppm}$ can be written as [7]:

$$
\frac{K(k)}{K\left(k^{\prime}\right)}=\frac{K(k)}{K^{\prime}(k)}\left\{\begin{array}{l}
\ln \left(2 \frac{1+\sqrt{k}}{1-\sqrt{k}}\right) / \pi \text { for } 0.5 \leq k^{2} \leq 1 \\
\pi / \ln \left(2 \frac{1+\sqrt{k^{\prime}}}{1-\sqrt{k^{\prime}}}\right) \text { for } 0 \leq k^{\prime 2} \leq 0.5
\end{array}\right.
$$

Wherek $=a / b, k^{\prime}=\sqrt{1-k^{2}}, k_{1}=\sinh (\pi a / 2 h) / \sinh (\pi b / 2 h), k_{1}^{\prime}=\sqrt{1-k_{1}^{2}}$

3.2.2. Coplanar Strips Model: Coplanar strips (CPS) are basically a coupled microstrip lines either symmetric or asymmetric, (two transmission lines) of width $\boldsymbol{W}_{\mathbf{1}}$ and $\boldsymbol{W}_{\mathbf{2}}$ on a dielectric substrate of finite thickness $\mathbf{h}$ separated by very small gap $\mathbf{s}$ as shown in Figure 3.

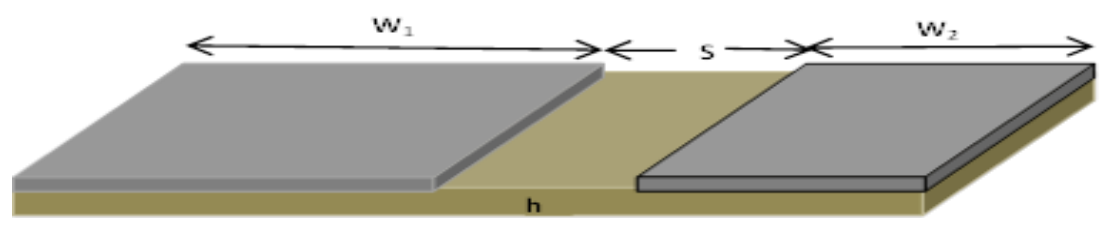

Figure 3. CPS Transmission Line Model of Width $W_{1}$ and $W_{2}$ Separated by Distance S Representing Equivalent Printed Folded Dipole Antenna

Characteristic impedance and effective dielectric constant of asymmetric CPS with finite substrate thickness is given by [7]:

$$
\begin{gathered}
Z_{c}=60 \pi K^{\prime}\left(k_{2}^{\prime}\right) /\left(K\left(k_{2}^{\prime}\right) \sqrt{\epsilon_{r e}}\right. \\
\epsilon_{r e}=1+q\left(\epsilon_{r}-1\right)
\end{gathered}
$$

With fill factor $=\mathbf{0 . 5}\left(\boldsymbol{K}\left(\boldsymbol{k}_{\mathbf{4}}\right) \boldsymbol{K}^{\prime}\left(\boldsymbol{k}_{2}^{\prime}\right)\right) /\left(\boldsymbol{K}^{\prime}\left(\boldsymbol{k}_{2}^{\prime}\right) \boldsymbol{K}\left(\boldsymbol{k}_{2}^{\prime}\right)\right)$, and also $k_{2}^{\prime}=\sqrt{\left(W_{1} W_{2}\right) /\left(W_{1}+S\right)\left(W_{2}+S\right)}, k_{4}=\sqrt{\frac{\sinh \left(\pi W_{1} / 2 h\right)}{\sinh \left(\pi\left(W_{1}+S\right) / 2 h\right)} \frac{\sinh \left(\pi W_{2} / 2 h\right)}{\sinh \left(\pi\left(W_{2}+S\right) / 2 h\right)}}$ 


\subsection{Effective Dielectric Constant of Even and Odd Mode}

The zero frequency approximation of effective permittivity $\left(\boldsymbol{\epsilon}_{\boldsymbol{r e}}\right)$ to account for the fringing can be expressed as [8]:

$$
\epsilon_{r e}(0)=0.5\left(\epsilon_{r}+1\right)+0.5\left(\epsilon_{r}-1\right)[1+10 / u]^{-A B}
$$

With $u=W / h, A=1+\ln \left[\left(u^{4}+\left(\frac{u}{52}\right)^{2}\right) /\left(u^{4}+0.432\right)\right] / 49+\left[\ln \left\{1+\left(\frac{u}{18.1}\right)^{3}\right\}\right] / 18.7$ and $\mathbf{B}=\mathbf{0 . 5 6 4}\left[\left(\epsilon_{r}-0.9\right) /\left(\epsilon_{r}+0.3\right)\right]^{0.053}$

The even-mode effective permittivity for coupled microstrip lines for $\mathbf{u}=\mathbf{W} / \mathbf{h}, \mathbf{g}=\mathbf{S} / \mathbf{h}$ and $\epsilon_{r}$ in the range $\mathbf{0 . 1} \leq W / \boldsymbol{h} \leq \mathbf{1 0}, \mathbf{0 . 1} \leq S / \boldsymbol{h} \leq \mathbf{1 0}$ and $\mathbf{1} \leq \boldsymbol{\epsilon}_{r} \leq \mathbf{1 8}$ for zero conductor thickness is given by [7]:

$$
\epsilon_{r e}^{\text {even }}(0)=0.5\left(\epsilon_{r}+1\right)+0.5\left(\epsilon_{r}-1\right)[1+10 / u]^{-A_{e}(v) B_{e}\left(\epsilon_{r}\right)}
$$

Where $\mathbf{v}=\mathbf{u}\left(\mathbf{2 0}-\boldsymbol{g}^{\mathbf{2}}\right) /\left(\mathbf{1 0}+\boldsymbol{g}^{2}\right)+\boldsymbol{g} \cdot \exp (-\boldsymbol{g})$,

$$
A_{e}(v)=1+\ln \left[\left(v^{4}+(v / 52)^{2}\right) /\left(v^{4}+0.432\right)\right] / 49+\ln \left[1+(v / 18.1)^{3}\right] / 18.7
$$

And $\boldsymbol{B}_{\boldsymbol{e}}\left(\epsilon_{r}\right)=\mathbf{0 . 5 6 4}\left[\left(\epsilon_{r}-\mathbf{0 . 9}\right) /\left(\epsilon_{r}+3\right)\right]^{0.058}$

The odd-mode effective dielectric constant for coupled microstrip lines for zero conductor thickness is given by [7]:

$$
\epsilon_{r e}^{\text {odd }}(0)=\left[0.5\left(\epsilon_{r}+1\right)+A_{o}\left(u, \epsilon_{r}\right)-\epsilon_{r e}(0)\right] \cdot \exp \left(-C_{0} g D_{0}\right)+\epsilon_{r e}(0)
$$

$$
\begin{gathered}
\boldsymbol{A}_{\boldsymbol{o}}\left(\boldsymbol{u}, \epsilon_{r}\right)=\mathbf{0 . 7 2 8 7}\left[\epsilon_{r e}(0)-0.5\left(\epsilon_{r}+1\right)\right] \cdot[1-\exp (-0.179 u)] \\
\boldsymbol{B}_{o}\left(\epsilon_{r}\right)=0.747 \epsilon_{r} /\left(\epsilon_{r}+0.15\right), C_{0}=B_{o}\left(\epsilon_{r}\right)-\left[B_{o}\left(\epsilon_{r}\right)-0.207\right] \cdot \exp (-0.414 u) \\
D_{0}=0.593+0.694 \cdot \exp (-0.562 u)
\end{gathered}
$$

\subsection{Characteristic Impedance of Even and Odd Mode}

The even-mode characteristic impedance of coupled microstrip lines is given by [7]:

$$
Z_{c e}(0)=Z_{0} \sqrt{\epsilon_{r e}(0) / \epsilon_{r e}^{\text {even }}(0)} /\left(1-\left[Z_{0}(0) / 377\right]\left[\epsilon_{r e}(0)\right]^{0.5} Q_{4}\right)
$$

With $Z_{0}(0)=60 / \sqrt{\epsilon_{\text {re }}} \ln \left[f(u) / u+\sqrt{1+(2 / u)^{2}}\right], \mathrm{u}=\mathrm{W} / \mathrm{h}$

$f(u)=6+(2 \pi-6) \exp \left[-(92 / 3 u)^{0.7528}\right], Q_{1}=0.8695 \cdot u^{0.194}, Q_{2}=1+0.7519 \mathrm{~g}+0.189$. $\mathrm{g}^{2.31}$

$Q_{3}=0.1975+\left[16.6+(8.4 / g)^{6}\right]^{-0.387}+\ln \left(g^{10} /\left[1+(g / 3.4)^{10}\right]\right) / 243$

$Q_{4}=\left(2 Q_{1} / Q_{2}\right) \cdot\left\{\exp (-\mathrm{g}) \cdot u^{Q_{3}}+[2-\exp (-\mathrm{g})] \cdot u^{-Q_{3}}\right\}^{-1}$

Similarly, the odd-mode characteristic impedance of coupled microstrip lines is [7]:

$$
\begin{aligned}
& Z_{c o}(0)=Z_{0} \sqrt{\epsilon_{r e}(0) / \epsilon_{r e}^{\text {odd }}(0)} /\left(1-\left[Z_{0}(0) / 377\right]\left[\epsilon_{r e}(0)\right]^{0.5} Q_{10}\right) \\
& Q_{5}=1.794+1 / 14 \cdot \ln \left[1+0.638 /\left(g+0.517 g^{2.43}\right)\right] \\
& \quad Q_{6}=0.2305+\ln \left[\left(g^{10} /\left(1+(g / 5.8)^{10}\right)\right] / 281.3+\ln \left(1+0.598 g^{1.154}\right) / 5.1\right. \\
& Q_{7}=\left(10+190 g^{2}\right) /\left(1+2.3 g^{3}\right) Q_{8}=\exp \left(-6.5-0.95 \ln (g)-(g / 0.15)^{5}\right)
\end{aligned}
$$




$$
Q_{9}=\ln \left(Q_{7}\right) \cdot\left(Q_{8}+1 / 16.5\right), \quad Q_{10}=\left[Q_{2} Q_{4}-Q_{5} \cdot \exp \left[\ln (u) \cdot Q_{6} \cdot u^{-Q_{9}}\right]\right] / Q_{2}
$$

\section{Simulation}

Considering the input impedance of the model in eqn. (1), it is possible to simulate and analyze the behavior of the model using eqn.(1a).-(1e),eqn.(2)-(3a) with eqn.(4) or eqn.(6). One of the important factors in the design of folded dipole antenna is its current division factor or impedance step up ratio $\alpha$ as given in eqn. (1a), eqn.(1b),eqn.(1c), eqn.(1d)and eqn.(1e), as their values determine the step up of impedance, depending upon the width and spacing between the two arms of the folded dipole antenna. Further, due to the coupling of the electromagnetic field between the coupled lines, the lines can support two propagation modes namely: (a) even and (b) odd modes [7]. Even-mode propagation implies two signals of the same amplitude and zero phase difference passing through the coupled line, while oddmode operation denotes the propagation of two signals of the same amplitude but $\mathbf{1 8 0}^{\mathbf{0}}$ phase difference [7]. Due to the inhomogeneous dielectric medium, namely: (i) air and (ii) substrate material, the two modes have different effective dielectric constants and phase velocities that degrade the performance of the coupled lines [7]. When operating at different modes, the effective capacitance between the two transmission lines and the ground plane is different and contribute to two impedances namely: (a) even-mode $\boldsymbol{Z}_{\boldsymbol{c o}}$ (eqn.(11)) and (b) odd-mode impedances $\boldsymbol{Z}_{\boldsymbol{c} \boldsymbol{e}}$ (eqn.(12)).The characteristic impedance of the coupled line dependents on $\boldsymbol{Z}_{\boldsymbol{c} \boldsymbol{e}}$ and $\boldsymbol{Z}_{\boldsymbol{c o s}}$. In order to evaluate the performance and accuracy of the model, we simulate it in MATLAB Platform. The Flow diagram of the simulation program is given in Figure 4.

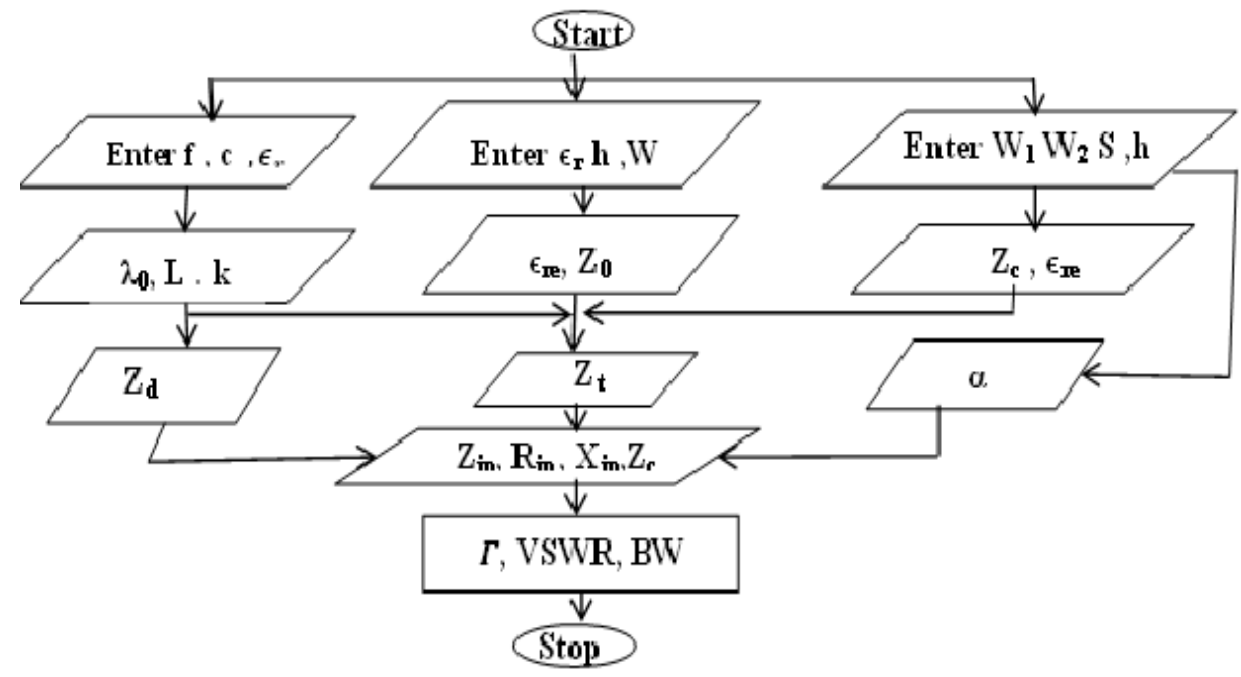

Figure 4. Flow Diagram of the Simulation Program

In the above simulations eqn.(1)-eqn.(12) are used to study various characteristics of the proposed asymmetric strip folded dipole antenna.

\section{Result and Discussions}

Real and imaginary part of input impedance as given in eqn.(1) of asymmetric and nearly symmetric strip folded dipole is simulated as a function of frequency with different values of 
$\boldsymbol{\alpha}$ obtained from eqn.(1a)-eqn.(1e) and the responses are shown in Figure 5-Figure 8 respectively. It is observed that the input impedance increases with frequency and the variation is uniform for nearly symmetric folded dipole antenna. At resonance frequency the input impedance is nearly four times that of single dipole. The current division factor $\boldsymbol{\alpha}$ obtained from eqn.(1a)-(1e) for asymmetric and nearly symmetric folded dipole is simulated as a function of dipole spacing and the responses are shown in Figure 9 and Figure 10 respectively. It is observed that for nearly symmetric folded antenna step-up impedance ratio is nearly equal when spacing is very small. The VSWR of eqn.(3a) using eqn.(1)eqn.(3) and eqn.(6) with different values of $\boldsymbol{\alpha}$, from eqn.(1a) -eqn.(1e) is simulated as a function of frequency and the responses are shown in Figure 11 and Figure 12 for asymmetric and nearly symmetric strip folded dipole antenna respectively. From the plot it is apparent that asymmetric folded dipole yield large Band Width (BW) with VSWR=2 compared to nearly symmetric folded dipole. We have simulated VSWR of eqn.(3a) using eqn.(1),eqn.(1a), eqn.(3) and eqn.(4) asymmetric folded dipole for waveguide mode while for CPS model we have used all the four equations but substituted value of characteristic impedance from eqn.(6) and the responses are shown in Figure 13 and Figure 14 respectively. The geometries such as, $\boldsymbol{W}_{\mathbf{1}}$, and $\boldsymbol{W}_{\mathbf{2}}$ are adjusted to tune the input impedance and to widen the BW. The VSWR of eqn.(3a) using eqn.(1)- eqn.(3) and eqn.(6) is simulated as function of line spacing $s$ for asymmetric and nearly symmetric folded dipole with different values of $\boldsymbol{\alpha}$ and responses are shown in Figure 15 and Figure 16 respectively. It is observed that for asymmetric folded dipole, current division factor $\boldsymbol{\alpha}$ described in eqn. (1b) yields $\boldsymbol{V S W R}>2$ when the spacing (s ) between the two arms of the folded dipole is increased, but other values of $\boldsymbol{\alpha}$ of eqn.(1a),(1c), (1d) and (1e) shows decrease in $\boldsymbol{V S W R}$, while for nearly symmetric folded dipole the variations of VSWR with spacing is almost linear. The values of design parameters are listed in Table 1-Table 5. The even-odd-mode effective dielectric constant and characteristic impedance as function of substrate height are simulated and the responses are depicted in Figure 17 and Figure 18 respectively. The even-mode effective dielectric constant and characteristic impedances are appeared to be more accurate within the substrate thickness range. The results show that the variation of characteristic impedance with substrate height is much sharper than the variation of an effective dielectric constant with substrate height. Further, it is seen that the even-mode parameters show a greater variation with substrate height than the odd mode parameters. The $\boldsymbol{\alpha}$ values of eqn.(1e) shown in Table-1 collapse giving real and imaginary part for nearly symmetric case when the element spacing between the folded dipole strips are very small. Further, when spacing $\mathbf{s}$ between the lines is very small the characteristic impedance and dielectric constant yields real and imaginary part as can be seen in Table 3. According to [12] the spacing act like a slot and it fails to resonate when the slot width is very small compared to widths of the coupled line. 


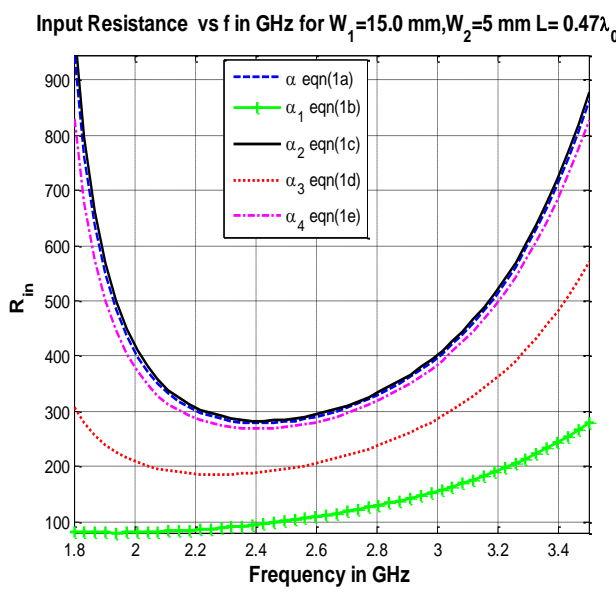

Figure $5 . \mathbf{R}_{\text {in }}$ as a Function of Frequency for an Asymmetric Folded Dipole

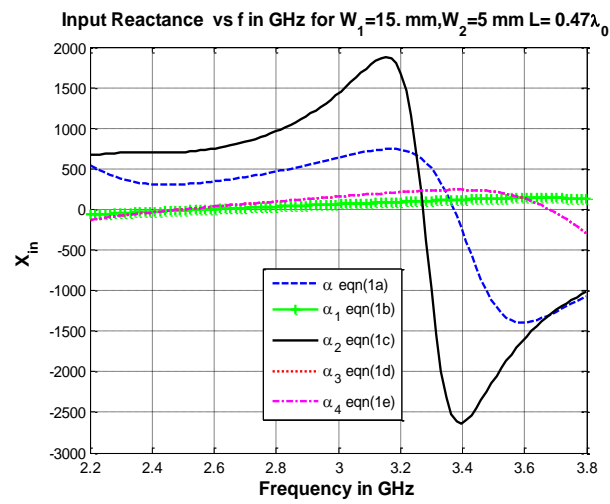

Figure 7. $X_{\text {in }}$ as a Function of Frequency for an Asymmetric Folded Dipole

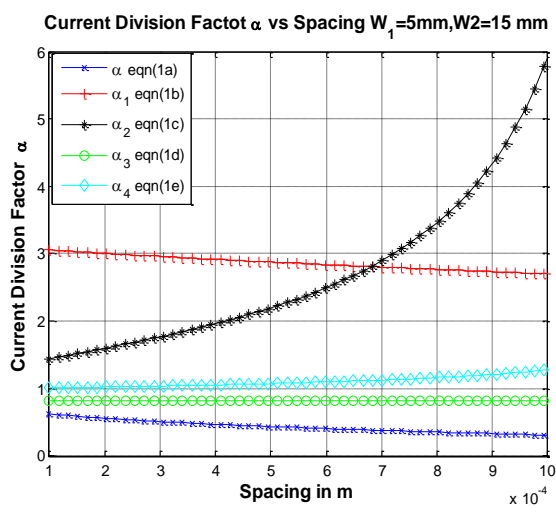

Figure 9. Current Division Factor a Function of Spacing for an Asymmetric Folded Dipole

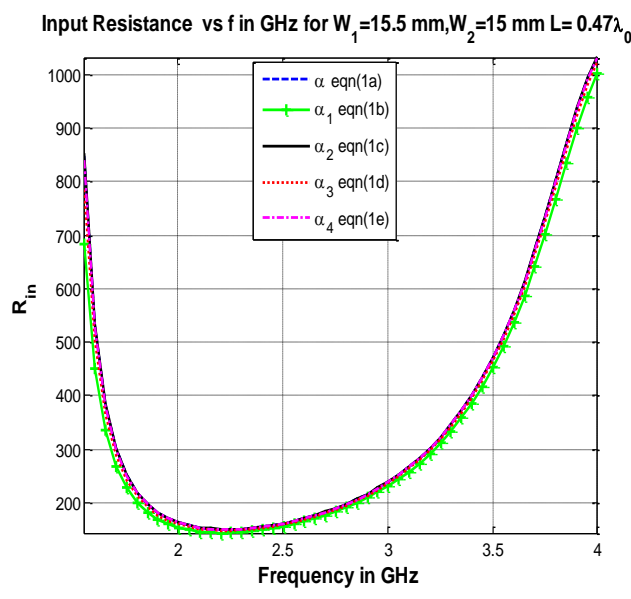

Figure $6 . R_{\text {in }}$ as a Function of Frequency for a Nearly Symmetric Folded Dipole

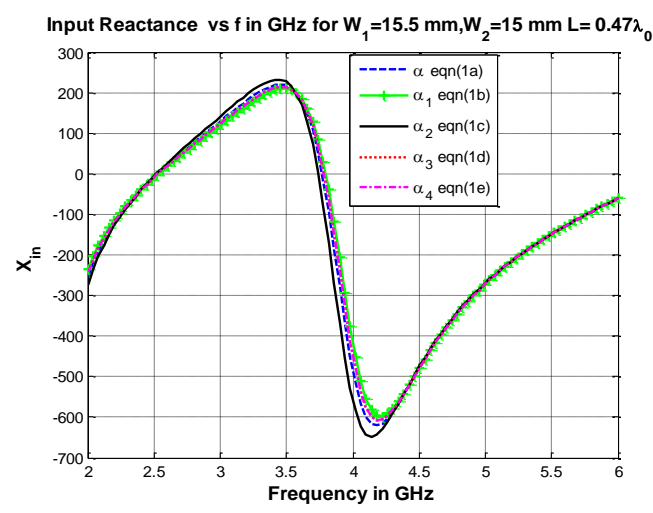

Figure 8. $X_{\text {in }}$ as a Function of Frequency for Nearly Symmetric Folded Dipole

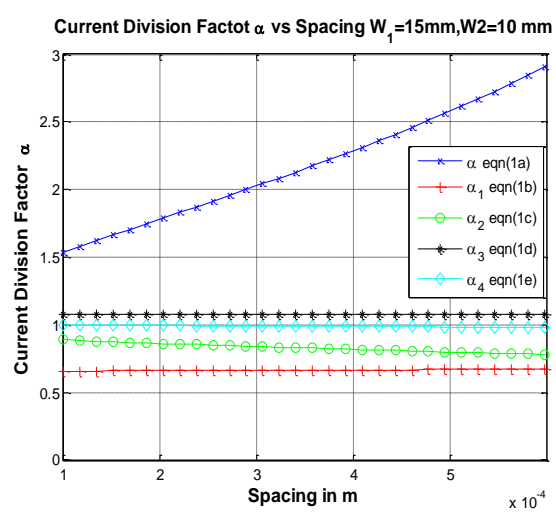

Figure 10. Current Division Factor as a Function of Spacing for a Nearly Symmetric Folded Dipole 


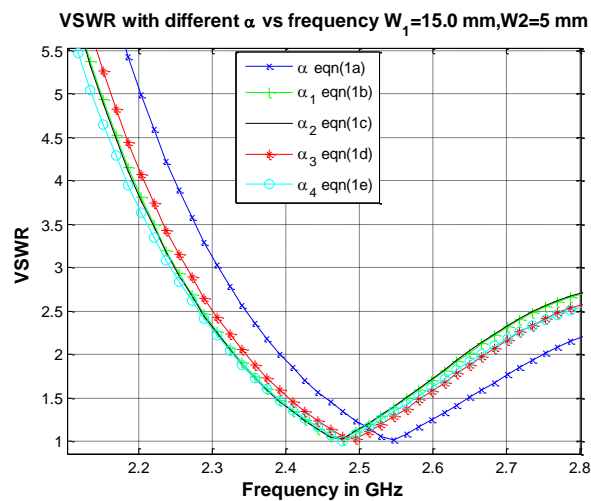

Figure 11. VSWR as a Function of Frequency for an Asymmetric Folded Dipole

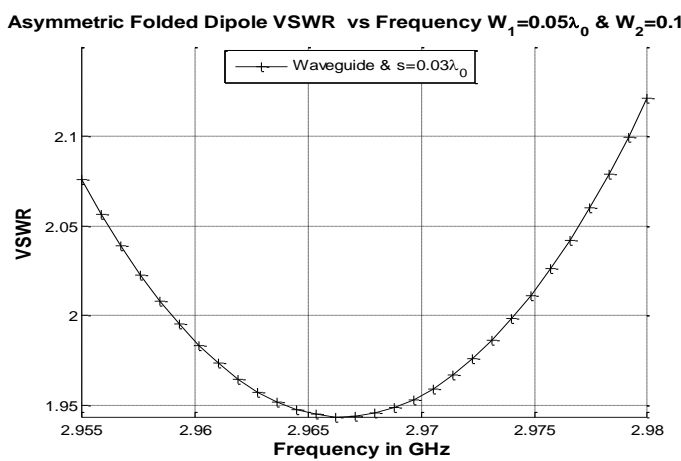

Figure 13. VSWR vs Frequency for a Modified Asymmetric Folded Dipole in Waveguide Model

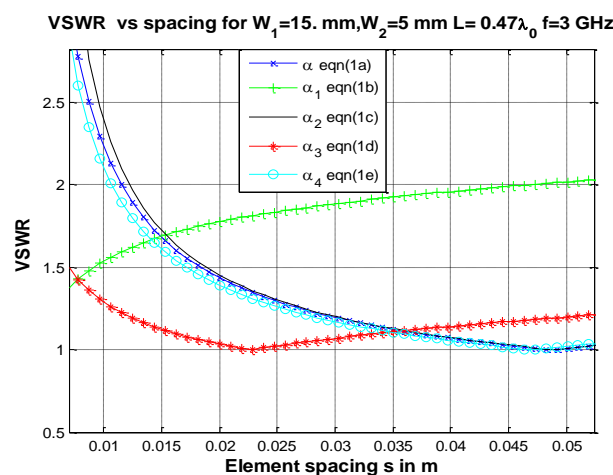

Figure 15. VSWR as a Function of Spacing (s) with Different Values of $\alpha$ for an Asymmetric Folded Dipole at $\mathrm{f}=3$ $\mathrm{GHz}$

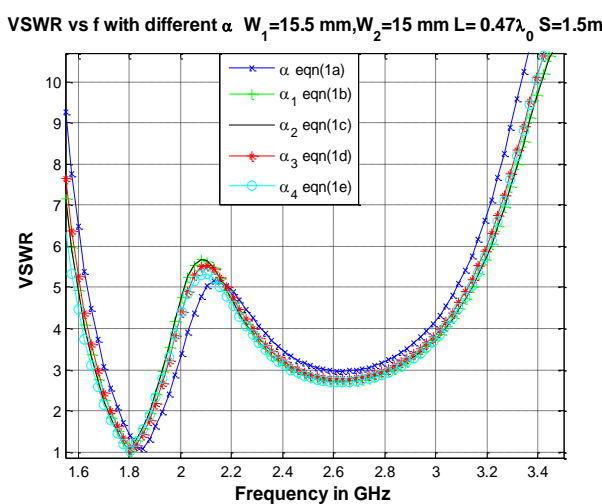

Figure 12. VSWR as a Function of Frequency for a Nearly Symmetric Folded Dipole

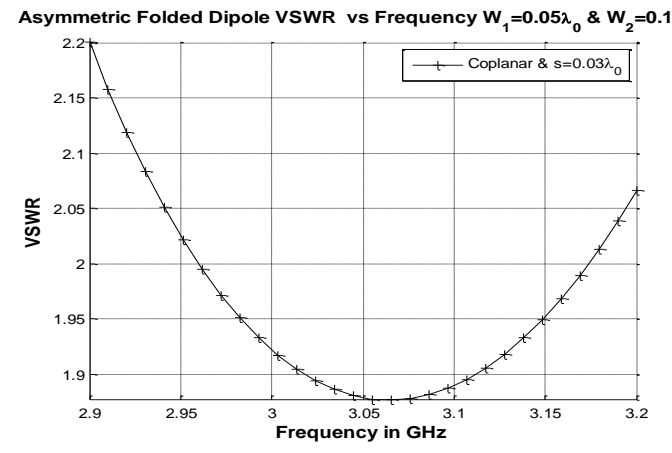

Figure 14. VSWR as a Function of $f$ (GHz) for an Asymmetric Folded Dipole in CPS Mode

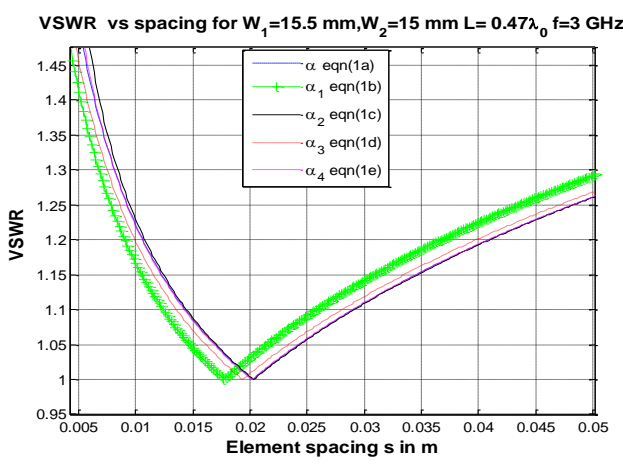

Figure 16. VSWR as a Function of $s$ (s> $0.05 \lambda_{0}$ ) with Different Values of $\alpha$ for Nearly Symmetric Folded Dipole at $\mathbf{f}=\mathbf{G H z}$ 
Table 1. Evaluation of Current Division Factor for $\epsilon_{r}=2.32, \mathrm{~h}=3 \mathrm{~mm}, \epsilon_{r e}=2.2$ $\& d=0.2 \mathrm{~mm}$

\begin{tabular}{|c|c|c|c|c|c|c|c|}
\hline s mm & $\begin{array}{l}W_{1} \\
\text { mm }\end{array}$ & $\begin{array}{l}W_{2} \\
\mathbf{m m}\end{array}$ & $\begin{array}{c}\alpha \\
\text { eqn.(1a) }\end{array}$ & $\begin{array}{c}\alpha_{1} \\
\text { eqn.(1b) }\end{array}$ & $\begin{array}{c}\alpha_{2} \\
\text { eqn.(1c) }\end{array}$ & $\begin{array}{c}\alpha_{3} \\
\text { eqn.(1d) }\end{array}$ & $\alpha_{4}$ eqn.(1e) \\
\hline 6.25 & 15 & 5 & 2.5323 & 0.5016 & 3.1507 & 1.2170 & 2.4094 \\
\hline 7.5 & 10 & 5 & 1.5655 & 0.6772 & 1.6309 & 1.1268 & 1.5129 \\
\hline 8.75 & 15 & 10 & 1.3853 & $\mathbf{0 . 7 7 3 3}$ & 1.4785 & 1.0800 & 1.3569 \\
\hline 11.25 & 20.5 & 20 & 1.0233 & 0.9833 & 1.0314 & 1.0052 & 1.0226 \\
\hline 20 & 15 & 5 & 1.6388 & 0.6165 & 1.6563 & 1.2160 & 1.5937 \\
\hline 21 & 10.5 & 10 & 1.0229 & 0.9780 & 1.0235 & 1.0090 & 1.0215 \\
\hline 2 & 15.5 & 15 & 1.0970 & 0.9707 & 0.9504 & 1.0065 & $0.9921+0.03 i$ \\
\hline 0.25 & 5.1 & 5 & 1.1453 & 0.9807 & 0.9878 & 1.0032 & $0.9993+.02 i$ \\
\hline
\end{tabular}

Table 2. Evaluation of Even-odd $M o d e Z_{o e}, Z_{o o}, \epsilon_{r e} \epsilon_{r e}^{e}, \epsilon_{r e}^{o}$, Coupled Line

\begin{tabular}{|c|c|c|c|c|c|c|c|c|}
\hline$\epsilon_{r}$ & h mm & S mm & W mm & $Z_{o e}$ ohms & $Z_{\text {oo }}$ ohms & $\epsilon_{r e}$ & $\epsilon_{r e}^{e}$ & $\epsilon_{r e}^{o}$ \\
\hline 2.32 & 3.1 & 2 & 5 & 73.38 & 54.75 & 1.884 & 1.887 & 1.571 \\
\hline 2.32 & 3.1 & 2 & 10 & 47.86 & 40.61 & 1.964 & 1.967 & 1.484 \\
\hline 3.7 & 2.5 & 1 & 5 & 52.28 & 37.47 & 2.853 & 2.880 & 2.276 \\
\hline 3.7 & 2.5 & 0.5 & 10 & 32.73 & 23.54 & 3.027 & $\mathbf{3 . 0 8 2}$ & 2.418 \\
\hline 6.15 & 1.9 & 0.5 & 5 & 35.12 & 24.63 & 4.64 & 4.73 & 3.59 \\
\hline 6.15 & 1.9 & 0.25 & 10 & 21.22 & 15.53 & 4.99 & 5.12 & 3.85 \\
\hline
\end{tabular}

Table 3. Evaluation of $Z_{o e}, Z_{o o}, \epsilon_{r e}, \in_{r e}^{c p s}$ Asymmetric Coplanar Strip Model

\begin{tabular}{|c|c|c|c|c|c|c|c|c|}
\hline$\epsilon_{r}$ & $\begin{array}{l}\mathbf{h} \\
\mathbf{m m} \\
\end{array}$ & S mm & $\begin{array}{l}W_{1} \\
(\mathbf{m m})\end{array}$ & $\begin{array}{l}W_{2} \\
(\mathbf{m m})\end{array}$ & $\epsilon_{r e}$ & $\epsilon_{\text {reI }}$ & $Z_{c e}$ & $Z_{c o}$ \\
\hline 2.32 & 3.1 & 1 & 5 & 10 & 1.924 & 1.257 & 130.29 & 132.30 \\
\hline 2.32 & 3.1 & 1 & 10 & 5 & 1.924 & $1.39+0.5 i$ & $118.3-2.1 i$ & $121.8+1.9 i$ \\
\hline 2.32 & 3.1 & 20 & 10 & 5 & 1.924 & 1.260 & 290.74 & 307.25 \\
\hline 3.7 & 2.5 & 20 & 5 & 10 & 2.943 & 1.517 & 265.09 & 301.62 \\
\hline 3.7 & 2.5 & 20 & 10 & 5 & 2.943 & 1.522 & 264.69 & 296.15 \\
\hline 6.15 & 1.9 & 20 & 5 & 10 & 4.823 & 1.984 & 231.79 & 289.87 \\
\hline 6.15 & 1.9 & 20 & 10 & 5 & 4.823 & 1.986 & 231.68 & 283.43 \\
\hline
\end{tabular}

Table 4. Evaluation of $Z_{o e}, Z_{o o}, \epsilon_{r e}, \epsilon_{r e}^{W G}$ Coplanar Waveguide Model

\begin{tabular}{|c|c|c|c|c|c|c|c|c|}
\hline$\epsilon_{r}$ & h mm & $\mathbf{S ~ m m}$ & $\begin{array}{c}W_{1} \\
(\mathbf{m m})\end{array}$ & $\begin{array}{c}W_{2} \\
(\mathbf{m m})\end{array}$ & $\epsilon_{r e}$ & $\epsilon_{\text {reI }}$ & $Z_{c e}$ & $Z_{c o}$ \\
\hline 2.32 & 3.1 & 1 & 5 & 10 & 1.924 & 1.070 & 34.42 & 34.99 \\
\hline 2.32 & 3.1 & 20 & 5 & 10 & 1.924 & 1.178 & 73.69 & 73.92 \\
\hline 3.7 & 2.5 & 1 & 5 & 10 & 2.943 & 1.133 & 33.45 & 34.16 \\
\hline 3.7 & 2.5 & 20 & 5 & 10 & 2.943 & 1.321 & 69.60 & 70.33 \\
\hline 6.15 & 1.9 & 1 & 5 & 10 & 4.823 & 1.236 & 32.02 & 33.09 \\
\hline 6.15 & 1.9 & 20 & 5 & 10 & 4.823 & 1.547 & 64.30 & 66.26 \\
\hline
\end{tabular}


Table 5. Calculation of BW for $\epsilon_{r}=2.32, W_{1}=15 \mathrm{~mm}, W_{2}=5 \mathrm{~mm}, \mathrm{~s}=10 \mathrm{~mm}$ and $\mathrm{L}=.5 \lambda_{0}$ with Different Values of $\alpha$ eqn.(1a)-eqn.(1e), Resonant Frequency $f_{r}$ for VSWR $\leq 2$ using Figure 12

\begin{tabular}{|l|l|l|l|l|l|l|l|l|}
\hline Eqn. No. & $\alpha$ value & $f_{r} \mathrm{GHz}$ & $\begin{array}{l}\mathbf{Z}_{\mathbf{c}} \text { ohms } \\
\text { eqn.(6) }\end{array}$ & $\mathbf{Z}_{\mathbf{t}} \mathbf{o h m s}$ & $\begin{array}{l}\mathbf{R}_{\text {in }} \\
\text { eqn.(2) }\end{array}$ & $\begin{array}{l}\text { Xin } \\
\text { eqn.(2) }\end{array}$ & $\begin{array}{l}\text { BW MHz } \\
\text { Fig.11 }\end{array}$ & BW\% \\
\hline Eqn.(1a) & $\mathbf{2 . 0 0 0 0}$ & $\mathbf{2 . 5 5}$ & $\mathbf{2 1 9}$ & $\mathbf{- 5 3 4 j}$ & $\mathbf{2 1 9}$ & $\mathbf{- 1 9 4 . 9 j}$ & $\mathbf{3 6 0}$ & $\mathbf{1 4 . 1}$ \\
\hline Eqn.(16) & $\mathbf{0 . 5 4 8 6}$ & $\mathbf{2 . 4 7 5}$ & $\mathbf{2 1 9}$ & $\mathbf{- 5 3 4 j}$ & $\mathbf{7 5 . 5}$ & $\mathbf{- 4 6 . 8 j}$ & $\mathbf{2 2 5}$ & $\mathbf{9 . 1}$ \\
\hline Eqn.(1c) & $\mathbf{2 . 1 2 0 1}$ & $\mathbf{2 . 4 7 5}$ & $\mathbf{2 1 9}$ & $\mathbf{- 5 3 4 j}$ & $\mathbf{2 2 9 . 9}$ & $\mathbf{- 2 1 1 . 5 j}$ & $\mathbf{3 0 0}$ & $\mathbf{1 2 . 1}$ \\
\hline Eqn.(1d) & $\mathbf{1 . 2 1 6 8}$ & $\mathbf{2 . 5}$ & $\mathbf{2 . 1 9}$ & $\mathbf{- 5 3 4 j}$ & $\mathbf{1 4 0 . 7}$ & $\mathbf{- 1 0 1 . 7 j}$ & $\mathbf{2 . 7 5}$ & $\mathbf{1 1}$ \\
\hline Eqn.(1e) & $\mathbf{1 . 8 9 9 5}$ & $\mathbf{2 . 4 7 5}$ & $\mathbf{2 . 1 9}$ & $\mathbf{- 5 3 4 j}$ & $\mathbf{2 0 9 . 5}$ & $\mathbf{- 1 0 1 . 7 j}$ & $\mathbf{3 0 0}$ & $\mathbf{1 2 . 1}$ \\
\hline
\end{tabular}

Figure 17. Even Odd Mode $\epsilon_{r e}$ as a Function of Height (h) for a Folded Dipole
Figure 18. Even-Odd Mode $Z_{0}$ eand $Z_{\mathrm{oo}}$ as a Function of $h$ for a Folded Dipole

\section{Conclusion}

A microstrip folded dipole antenna is modeled and simulated with center frequency of $3 \mathrm{GHz}$ and fractional BW is determined for VSWR $\leq \mathbf{2}$. It is observed that the folded dipole design gives wide impedance BW with variation of spacing and width ratio of folded arms. The odd-even mode design is applied to calculate input impedance and effective dielectric constant for asymmetric strip folded dipole. The values of current division factor $(\boldsymbol{\alpha})$ proposed in five models are compared. It differs significantly from the original folded dipole antenna model (eqn.1a) of circular cross section transformed to corresponding strip dipole model. The simulation is performed with $\boldsymbol{\alpha}$ of different models. The results closely agree for nearly symmetric folded dipole antenna and BW values are almost equal. In case of asymmetric folded dipole, the maximum value of BW is obtained; when $\boldsymbol{\alpha}$ of eqn. (1a) is used, while eqn. (1b) yields minimum value of BW. However if the width of center conductor $\mathbf{W}_{\mathbf{1}}$ is made smaller than the width of folded arm $\mathbf{W}_{\mathbf{2}}$, eqn. (1b) yields large BW while all other values of $\boldsymbol{\alpha}$ (eqn. (1a), (1c)-(1e)) gives smaller BW when $\mathbf{W}_{1}<\mathbf{W}_{2}$.

\section{Acknowledgement}

Authors are thankful to the Head, Department of Electronics and Communication Technology, Gauhati University for providing valuable suggestions and necessary computing facilities at the laboratory during the work. 


\section{References}

[1] D. Edward and D. Rees, “A Broadband Printed Dipole with Integrated Balun”, Microwave Journal, vol. 30, no. 5, (1987).

[2] G. A. Thiele, E. P. Ekelman, Jr., and L. W. Henderson, "On the Accuracy of the Transmission Line Model for Folded Dipole”, IEEE Trans. Antennas Propagate., vol. AP-28, no. 5, (1980), pp. 700-703.

[3] R. W. Lampe, "Design Formulas for an Asymmetric Coplanar Strip Folded Dipole", IEEE Trans. Antennas. Propagation, vol. AP-33, no. 9, (1985), pp. 1028-1031.

[4] R. W. Lampe, "Corrections to, Design Formulas for an Asymmetric Coplanar Strip Folded Dipole", IEEE Trans. Antennas. Propagation, vol. AP-34, no. 4, (1986), p. 611.

[5] H. J. Visser, "Improved Design Equations of Asymmetric Coplanar Strip Folded Dipoles on a Dielectric", EUCAPIEEE Xplore, (2010), pp.1-6.

[6] G. Hua, C. Yang, P. Lu, H.-X. Zhou, and W. Hong, "Microstrip Folded Dipole Antenna for 35 GHz MMW Communication", Hindawi Publishing Corporation, International Journal of Antennas and Propagation, vol. 2013, Article ID 603654, 6 pages (2013).

[7] R. Garg, P. Bhartia, I. Bahl and A. Ittipiboon, "Microstrip Antenna Design Handbook", Artech House, Norwood, MA, (2001), pp. 399-810.

[8] G. Kumar and K. P. Ray, "Broadband Microstrip Antenna”, Artech House, (2003), pp. 383-388.

[9] C. A. Balanis, "Antenna Theory: Analysis and Design: Wiley-Inter science", Hoboken, New Jersey, USA, (2005), pp. 514-533.

[10] T. A. Milligan, "Modern antenna design", 2nd edition John Wiley \& Sons, Hoboken, New Jersey, (2005), pp. 330-334.

[11] J. K. Raines, "Folded Unipole Antennas Theory and Applications", McGraw-Hill, New York, (2007), p. 239.

[12] C.-T. Tai and S. A. Long, "in Antenna Engineering Handbook", Edited John L. Volakis McGraw-Hill (www.digitalengineeringlibrary.com), Vol. 4,pp.14-16,(2007).

\section{Authors}

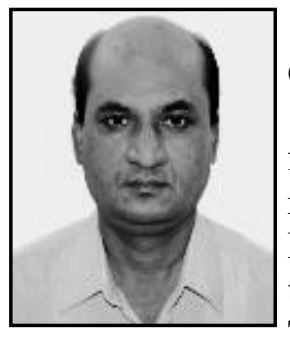

Tulshi Bezboruah, he received the B.Sc. degree in physics with electronics under the University of Dibrugarh, Dibrugarh, India, in 1990, and the M.Sc. and Ph.D. degrees in electronics and radio physics from Gauhati University, Guwahati, India, in 1993 and 1999, respectively. In February 2000, he joined in the Department of Electronics Science, Gauhati University, as a Lecturer. He is currently the Professor \& Head, Department of Electronics and Communication Technology, Gauhati University. His current research interest include web based instrumentation and control, web service, and communication systems. Professor Bezboruah is member of the IEEE, IEEE Geoscience and Remote Sensing Society as well as an Associate Member of the International Center for Theoretical Physics, Trieste, Italy.

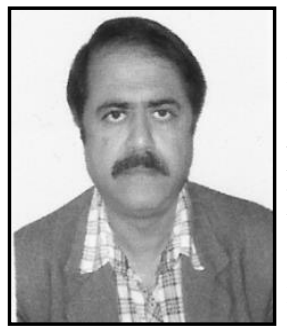

Prabir Banerjee, he received the BSc degree with honors in Physics under Gauhati University, Guwahati, India in 1984, and M. Sc (Electronics) degree from Delhi University in 1988. In November 1989 he joined Department of Physics, Jagiroad College, Jagiroad, Morigaon. Assam, India as a Lecturer, he is currently holding the post of Associate Professor in Physics in the same college. His research interest includes Microstrip Antennas and communication technology 
International Journal of Hybrid Information Technology Vol. 7, No. 6 (2014) 